Interfaces

\title{
Illustrer « les sentiers de la création » : des cheminements singuliers
}

\section{Alice Scheer}

\section{(2) OpenEdition}

1 Journals

Édition électronique

URL : http://journals.openedition.org/interfaces/665

DOI : 10.4000/interfaces.665

ISSN : 2647-6754

Éditeur :

Université de Bourgogne, Université de Paris, College of the Holy Cross

\section{Édition imprimée}

Date de publication : 12 décembre 2019

Pagination : 7-24

ISSN : 1164-6225

\section{Référence électronique}

Alice Scheer, «Illustrer « les sentiers de la création » : des cheminements singuliers », Interfaces [En ligne], 42 | 2019, mis en ligne le 12 décembre 2019, consulté le 06 janvier 2021. URL : http:// journals.openedition.org/interfaces/665; DOI : https://doi.org/10.4000/interfaces.665

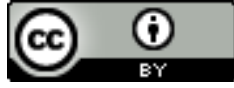

Les contenus de la revue Interfaces sont mis à disposition selon les termes de la Licence Creative Commons Attribution 4.0 International. 


\title{
ILLUSTRER «LES SENTIERS DE LA CRÉATION » : DES CHEMINEMENTS SINGULIERS
}

\author{
Alice Scheer \\ Université Lyon 2
}

\section{Résumé}

La collection des « sentiers de la création », publiée par Albert Skira et Gaëtan Picon entre 1969 et 1976, a pour principe éditorial d'être illustrée par les auteurs euxmêmes. Qu'ils aient réalisé leurs propres illustrations (comme Ionesco, Char ou Michaux par exemple) ou qu'ils se soient servis d'images déjà existantes, c'est avec une grande liberté que les auteurs se sont emparés de cette commande. Surprenante, l'iconographie témoigne parfois d'une certaine insubordination de l'image au texte qu'elle est supposée illustrer et manifeste entre eux un décalage. Cette étude interroge la manière singulière dont l'image s'associe aux mots et devient, pour ces écrivains, un moyen d'expression complémentaire pour penser et représenter la création. Elle s'appuie ainsi sur les ouvrages de J. M. G Le Clézio, Jean Tardieu, Octavio Paz, Claude Simon, Roland Barthes, et Yves Bonnefoy.

\begin{abstract}
The books published within the "sentiers de la création", a book series released by the Genevan publisher Albert Skira and the French writer Gaëtan Picon, are singular because they were illustrated by the authors themselves. While some of the latter have made their own images (as Ionesco, Char or Michaux for instance), others submitted a list of pictures they had taken from other artists and other times. All of them interpreted Skira's request very freely and the reader can sometimes notice a slight discrepancy between the image and the text. This article addresses the way the images associate with words to become, for the writers, a new and complementary medium to reflect on their own creation and represent it. It is based on the books written by J. M. G Le Clézio, Jean Tardieu, Octavio Paz, Claude Simon, Roland Barthes and Yves Bonnefoy.
\end{abstract}


« Les sentiers de la création », collection parue entre 1969 et 1976 aux éditions d'art Albert Skira et co-dirigée par Gaëtan Picon, réunit des ouvrages de commande singuliers car ils ont été illustrés par leurs auteurs. Là résidaient le principe et l'originalité mêmes d'une collection qui invitait les auteurs à revenir sur leur parcours créateur en mêlant les mots et les images. Écrivains, artistes et penseurs aussi divers qu'Aragon, Jean Dubuffet, Yves Bonnefoy, Joan Miró, Francis Ponge, Henri Michaux, ou Claude Lévi-Strauss, pour n'en citer que quelques-uns, devaient donc fournir, au moment de la remise du manuscrit, une liste d'une trentaine d'illustrations et participaient ensuite à l'élaboration de la maquette, en étroite collaboration avec l'équipe éditoriale. Dans ces ouvrages, l'iconographie devient alors un moyen d'expression complémentaire et c'est dans un double cheminement, visuel et textuel, que les auteurs pensent la création et s'efforcent de la représenter.

Si l'illustration explicative et subordonnée au texte est récurrente, notamment dans les carnets ou les essais consacrés à l'art ${ }^{1}$, c'est pourtant une certaine insubordination de l'image au texte qui, souvent, nous arrête. L'image surprend au fil de la lecture, paraît gagner en " autonomie » et il semble se dessiner parfois un écart entre l'image et le propos développé dans le texte. Pourquoi ces auteurs choisissent-ils de privilégier et de manifester ainsi l'écart ou le décalage entre le texte et l'image ? Il semble que ces écarts et décalages aient, dans " les sentiers de la création », trois enjeux différents. Le passage du texte à l'image permet d'abord de manifester un profond désir d'altérité et il suggère alors la volonté de l'auteur de "s'écarter de lui-même ». Pour certains auteurs, cet écart est ensuite un moyen de rendre compte des tâtonnements, des tours et des détours d'une création « en train de se faire ». Enfin, il peut participer plus directement d'une pensée de l'auteur sur la création comme écart, vacillement ou appréhension du vide.

\section{L'illustration comme altérité}

\section{S'écarter de soi, nécessaire et impossible [Haï, Le Clézio]}

Pour Le Clézio, qui consacre son ouvrage aux Indiens du Panama chez lesquels il s'est rendu, il est nécessaire et pourtant fondamentalement impossible de s'écarter de soi pour se tourner vers l'Autre. Dès les première pages de Haï, il présente en effet la tension inhérente à son projet de la façon suivante : « ces pages écrites pour parler de gens dont la grande vertu est d'être inaccessibles et

On peut ainsi penser à « La Chute d'Icare » de Picasso (1971), aux Mots dans la peinture de Michel Butor (1969) ou encore aux Carnets catalans de Joan Miró (1976). 
silencieux, ne savent parler, malheureusement, que de leur auteur » (Le Clézio 8). Ainsi, Le Clézio souligne tantôt l'opposition, tantôt l'analogie entre " Eux », les indiens Embera, et " Nous », les occidentaux. Les illustrations et les relations entre texte et images manifestent et interrogent cette opposition constante entre mouvement vers l'autre et retour à soi. À la fin de l'ouvrage, la table des illustrations classe d'ailleurs les images en deux groupes : pour « Nous » ce sont des photographies et des pages publicitaires arrachées à des magazines ; pour «Eux » ce sont des objets indiens, « vivants », magiques et « périssables » : paniers et colliers tressés, bois gravés etc.

Dans le chapitre «Kakwahaï, Le corps exorcisé », la tension entre texte et illustration manifeste simultanément l'écart et la proximité entre « occidentaux » et « indiens du panama ». Ces pages évoquent la peinture corporelle et éphémère pratiquée par les indiens à l'aide du suc d'un fruit, le genippa.

En s'effaçant avec le temps, avec l'eau de la pluie et l'eau des fleuves, la peinture corporelle oblige à reconnaître à chaque fois ce miracle de la peau, membrane transparente où passent les signes de la vie intérieure, fragile, tendre pellicule qui demande qu'on veille sur elle, qu'on la protège, qu'on la cache, qu'on défende ses secrets. (138)

La peau constitue donc une frontière poreuse entre les mondes intérieur et extérieur. Sur la double-page qui précède cette citation (136-137), la maquette du livre rapproche trois reproductions autour d'un motif récurrent : le cœur dessiné. À gauche de la première page se trouve en effet une publicité pour une bouteille de Perrier sur la condensation de laquelle un cœur a été dessiné au doigt. La page de droite reproduit une photographie de profil du « Vieux Léon », vieil indien Embera, prise par Marina Le Clézio. L'homme porte un chapeau et un fusil et on distingue, parmi les traits qu'il a tracés sur son visage, un cœur peint sur sa joue droite. Au centre enfin, une publicité pour des radiateurs en fonte semble faire le lien entre les deux images précédentes. Deux cœurs y sont dessinés à la craie et percés ensemble par une flèche imitant grossièrement celle des indiens. Ainsi, tandis que l'occidental peint exclusivement sur des surfaces autres, distinctes de lui-même, l'indien, lui, peint sur son propre corps. Pour Le Clézio, cette peinture corporelle manifeste simultanément la conscience de soi et celle de l'autre :

L'indien peint sa peau, et il cesse d'être nu. Sa peau devient toute pareille à un miroir, qui ne renvoie aux yeux des ennemis que leur propre image. Et par chacun des dessins écrits, au même moment la peau se met à voir, miracle, elle se couvre de milliers d'yeux ! (135)

En effet, peindre sur sa peau c'est la rendre voyante, capable de voir, visible et pourtant invisible aux yeux des autres ; c'est peindre à la fois pour protéger le corps des agressions du monde extérieur 
et pour se préserver du regard des autres, renvoyés, lorsqu'ils l'observent, à leurs propres corps et conscience. Les dessins publicitaires, certes eux aussi éphémères, paraissent dérisoires en comparaison du rite accompli par les indiens, évoqué dans le texte. Pourtant, si Le Clézio a choisi ces images de notre monde et refusé pour son ouvrage toute reproduction de l'art occidental, muséifié, fossilisé, c'est peut-être précisément parce qu'elles sont plus proches de l'art essentiel des peuples indiens qui ne cherche pas à résister au passage du temps. Ces deux types de dessins éphémères paraissent donc à la fois similaires et fondamentalement opposés. Le travail d'illustration transpose ainsi l'hésitation entre opposition et analogie, signe d'une impossibilité de parler des autres sans parler de soi.

\section{Quand l'écriture jalouse le visuel : Obscurité du jour de Jean Tardieu.}

Mais dans la collection, l' "Autre » que cherchent à approcher un certain nombre d'auteurs est avant tout « un autre moyen d'expression ». L'écriture de Jean Tardieu, par exemple, jalouse les moyens de la peinture et deux d'entre eux tout particulièrement : son immédiate expressivité et sa capacité à se défaire du sens. C'est pourquoi, les relations entre texte et image témoignent parfois des tentatives d'abolition de la frontière entre lisible et visible. On le voit notamment dans les reproductions d'une Calligraphie dédiée à un moineau mort d'Ikkyu Sojun (Tardieu 63), d'un tableau de Jean Cortot intitulé Écritures (64) datant de 1974 et figurant une langue imaginaire, ou encore dans le « poème à voir » de Jean Tardieu ayant pour titre Paysage (68-69) (fig. 1).

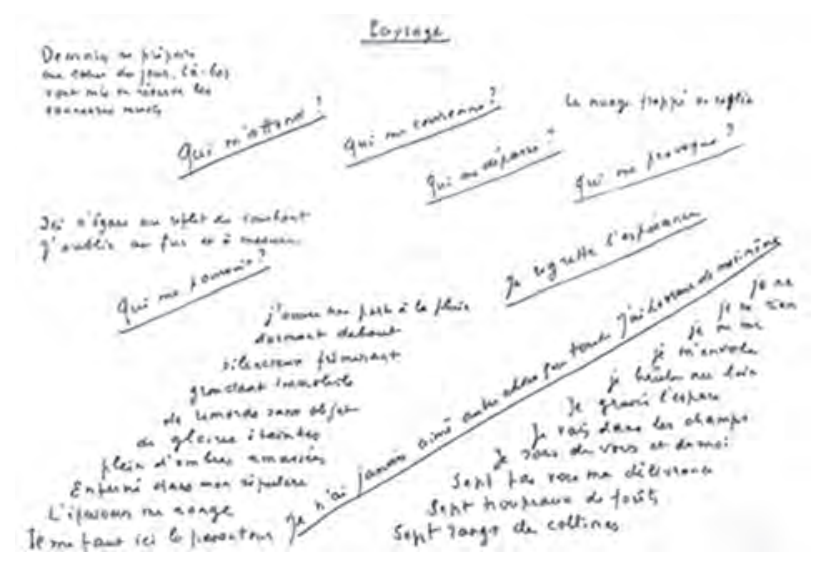

Ce dernier est placé sur une double page et les phrases inscrites horizontalement figurent les éléments du paysage en même temps qu'elles rendent compte de l'expérience du sujet au sein de ce décor. Le ciel est évoqué dans les coins supérieurs droit et gauche du tableau, soit à l'endroit même où le peintre le placerait sur sa toile : « demain se prépare au cœur

Fig. 1. TARDIEU, Jean. « Paysage ». Reproduction d'une double page d'Obscurité du jour. Les sentiers de la création. Genève : Albert Skira, 1972. 68-69. (C) Éditions Albert Skira 
du jour », « là-bas sont mis en réserve les tonnerres muets », " le nuage frappé se replie ». Un peu plus bas, au niveau de l'habituelle ligne d'horizon dans le deuxième tiers supérieur du tableau, Tardieu évoque les jeux de lumière d'un soleil couchant : " Ici s'égare un reflet du couchant ». En bas, à droite, ce sont « sept troupeaux de forêts » et «sept rangs de collines » qui sont ainsi imaginées. A ces divers éléments de description se mêlent les impressions d'un sujet observateur interrogeant sa place au cœur du paysage et, plus généralement sans doute, dans le monde. En effet, à la manière de lignes de fuite, des interrogations ou exclamations désespérées traversent ce « Paysage » de part en part, diagonalement : " Qui m'attend ? », "Qui me dépasse ? », " Je regrette l'espérance ». Dans ce poème visuel, l'écriture est donc simultanément visible et lisible, voire visible parce que lisible. Tardieu fait appel à l'expérience visuelle du lecteur et convoque, à travers ses souvenirs d'amateur d'art, d'autres paysages peints. Mais l'écriture permet encore d'intégrer ce que le tableau ne montre que plus difficilement : l'impression de temps vécu. Temporalité du paysage et temporalité de la mémoire se confondent. En effet, tandis que le reflet du couchant «s'égare », le sujet « oublie au fur et à mesure »; comme la pluie à laquelle il « ouvre une porte » et qu'il semble contempler, le sujet demeure « silencieux frémissant / grondant immobile /de remords sans objets/ de gloires éteintes / plein d'ombres amassées ». Dans " Paysage », l'écriture décrit en même temps qu'elle dérobe le visible, toujours approché sans être jamais fixé. Elle permet ainsi de rendre compte de l'expérience paradoxale que fait le poète du monde environnant car elle s'apparente à une approche simultanée de l'apparition et de la disparition, car elle donne à voir le plein en même temps que le vide. Les négations abondent et prennent une valeur absolue : « je ne », « je ne rien », « je ne me ». Chaque ajout, chaque nouvelle touche conduit en fait à une négation supplémentaire. «L'épaisseur me ronge », écrit ainsi Tardieu dans le coin inférieur gauche de son « poème à voir », comme si l'accumulation était en fait une altération, une désagrégation. Noircissant la page blanche de lignes obliques qui paraissent biffer un paysage naissant aux yeux du lecteur-observateur, l'écriture défait l'unité du sujet.

Dans cet ouvrage, l'illustration n'a donc pas pour seul but de montrer l'idéal pictural vers lequel tend l'écriture : elle constitue également un moyen d'expression complémentaire permettant à Jean Tardieu de partir en quête de ce qu'il nomme « l'Anti-Sens ». L'Anti-Sens n'est pas une absence de sens mais l'expression d'un refus du sens unique, d'une clôture du sens. C'est par conséquent, tout à l'inverse, une multiplication des sens possibles. Le premier chapitre de l'ouvrage a pour titre « le sens dans tous les sens » et le texte repose sur quantités de paradoxes, d'apparentes contradictions, de « nonsense » et d'ambiguïtés que l'oxymore du titre obscurité du jour soulignait déjà. D'autres images, comme L'Empire des lumières de Magritte (17), appuient, à leur manière, cette réflexion menée sur la contradiction et le paradoxe pour échapper à l'univocité du sens. L'iconographie d'Obscurité du jour 
complexifie et sature ainsi le sens. Elle est un nouveau moyen pour « chercher ce qui se résout dans sa contradiction » (101). Texte et image figurent ensemble mais aussi indépendamment la réversibilité du langage et des choses ${ }^{2}$.

\section{Les sentiers de l'illustration : montrer la création en train de se faire.}

Le décalage entre texte et image peut aussi constituer un moyen de représenter la création en train de se faire, les tâtonnements, les tours et détours de la création. La portée de ces décalages devient alors plus réflexive encore.

\section{Octavio Paz, Le Singe grammairien : création et analogie.}

Le lecteur du Singe grammairien d'Octavio Paz peut, au fil des images, suivre le cheminement réel et intellectuel de l'écrivain et du dieu singe Hanuman en Inde, sur le sentier de Galta au Rajasthan. On distingue deux types d'illustrations dans cet ouvrage : celle qui figure le chemin lui-même ainsi que le temple hindou de Galta, et celle qui témoigne des réflexions et des rêveries du marcheur à propos de la création. La première série d'images - des photographies du sentier, de pèlerins, de la façade du palais de Galta, des cours intérieures du sanctuaire ainsi que des singes qui les peuplent, toutes prises par Eusebio Rojas Guzmán, secrétaire de l'auteur - rythme la progression de l'ouvrage. C'est particulièrement le rôle conféré à la photographie de la façade principale du palais, dont différents tirages sont reproduits à plusieurs reprises dans l'ouvrage. Ces images, qui renvoient à un cheminement circonstanciel et géographiquement situable, côtoient des reproductions d'origines très diverses, empruntées aussi bien à des peintres tels qu'Henri Michaux, Max Ernst, John Constable, Richard Hamilton, Jean Dubuffet ou encore Francis Bacon, qu'au patrimoine hindou (sculptures du dieu singe Hanuman, miniatures tirées du Râmâyana...). Ces deux « lignes » d'illustration s'entrecroisent tout au long du livre et reviennent, in fine à Galta.

2 Voir à ce propos et pour plus de précision, un article que j'ai consacré pour la revue en ligne Textimage à l'illustration de l'ouvrage de Jean Tardieu: Scheer, Alice. « La part des images ou le saisissement de la contradiction dans Obscurité du jour de Jean Tardieu », Textimage, Varia 5, Printemps 2016. https://www.revue-textimage.com/12_ varia_5/scheer3.html. 


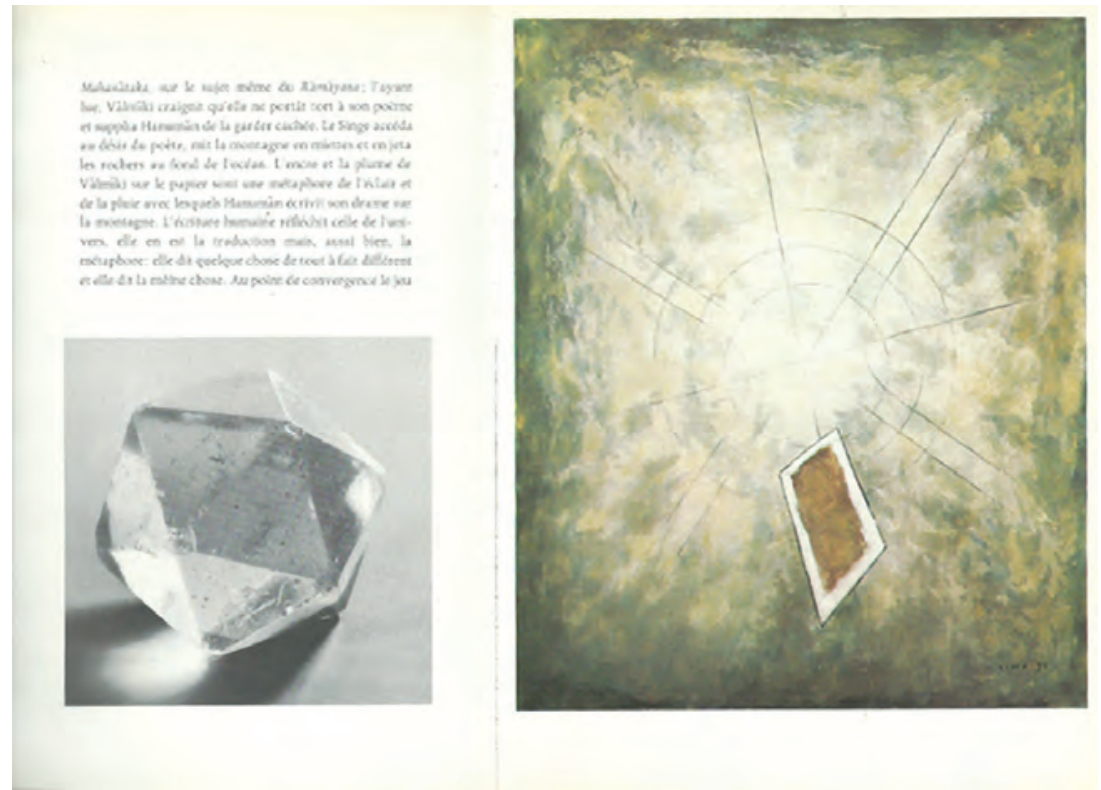

Fig. 2. À gauche, Tétraèdre de cristal- Tibet, XVIIIe siècle, Collection John Dugger et David Medalla, Londres. (Photo Arts Council of Great Britain, Londres) (Tous droits réservés). Dans PAZ, Octavio. Le Singe grammairien. Les sentiers de la création. Genève : Albert Skira, 1972. 154. (C) Éditions Albert Skira. À droite, SIMA, Joseph. Le point Un, 1970. Huile sur toile. $(65$ x 54). Genève : collection Edwin Engelberts. (Photo Maurice Babey, Bâle). Dans PAZ, Octavio. Le Singe grammairien. Les sentiers de la création. Genève : Albert Skira, 1972. 155. (C) Adagp, Paris, 2019 .

Si l'on se concentre sur la seconde de ces deux « lignes », on remarque qu'elle compose des réseaux par similitudes de formes. La maquette de l'avant-dernier chapitre du livre (Paz 154-157) est à ce titre révélatrice : elle crée un diptyque à partir d'un tétraèdre en cristal du Tibet et d'une huile de Joseph Sima intitulée Le point Un (fig. 2). L'œil du lecteur-observateur retrouve aisément dans la toile de Sima les formes triangulaires et les arêtes du tétraèdre. Ce diptyque est ensuite prolongé, à la page suivante, par la photographie d'un autre minéral : une stibnite provenant d'une mine de Baia Sprie, en Roumanie (fig. 3). Cette dernière réunit à la fois les faisceaux issus d'un point central, que l'on trouve dans la peinture de Sima, mais qui semblent ici démultipliés, et les formes géométriques et minérales du tétraèdre. Ces rapprochements formels consacrent la création par analogies qu'Octavio Paz évoque par ailleurs dans le texte accompagnant ces images : 
En abordant ces pages j'avais décidé de suivre à la lettre la métaphore du titre de la collection à laquelle elles sont destinées, Les sentiers de la création, et j’avais pensé écrire, tracer un texte qui effectivement fût un chemin et qui pût être lu, parcouru en tant que tel. À mesure que j'écrivais, le chemin de Galta s'effaçait ou bien je m'égarais et me perdais en ses défilés. À plusieurs reprises il me fallut revenir à mon point de départ. Au lieu d'avancer, le texte tournait sur lui-même.

La destruction est-elle création ? Je ne sais, mais je sais que la création est destruction. À chaque tournant le texte se dédoublait en un autre, à la fois sa traduction et sa transposition : une spirale de répétitions et de réitérations qui ont abouti à la négation de l'écriture comme chemin. Je me rends compte à présent que mon texte n'allait nulle part, sinon à la rencontre de soi-même. Je remarque également que les répétitions sont des métaphores et que les réitérations sont des analogies : un système de miroirs qui, peu à peu, ont révélé un autre texte. (156)

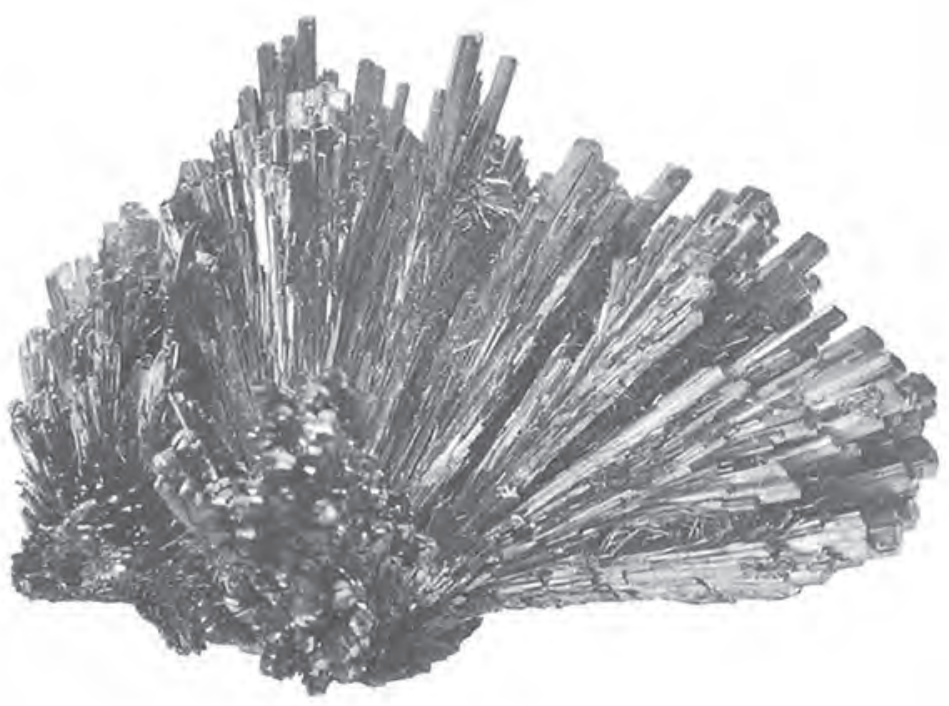

Fig. 3. Stibnite provenant de Baia Sprie. Collection privée, Genève (Photo Maurice Babey, Bâle). Dans Paz, Octavio. Le Singe grammairien. Les sentiers de la création. Genève : Albert Skira, 1972. 157. (C) Éditions Albert Skira (tous droits réservés). 
Parce que l'écriture ne saurait s'apparenter à une progression sur un chemin direct, dont elle ne peut que détruire l'unité en multipliant écarts et détours, ces trois illustrations, plus analogiques qu'explicatives, rappellent elles-mêmes d'autres images du livre. Ensemble, elles composent un « système de miroirs » : les faisceaux présents dans le tableau de Joseph Sima ainsi que dans la stibnite de Baia se retrouvent dans une photographie de Maria-José Paz prise par l'auteur à l'observatoire de Jaipur (100), mais aussi dans une lithographie d'Odilon Redon, intitulée Vision (113) 3 .

Moins que d'une illustration asservie, soumise au texte, il s'agit donc plutôt, dans Le Singe grammairien d'Octavio Paz, d'une illustration qui explore le caractère analogique de la création, invitant peut-être le lecteur à imiter le cheminement de l'écrivain sur les sentiers de la création en quittant, à son tour, le fil de la lecture pour revenir sans cesse à d'autres chapitres et à d'autres illustrations.

\section{Claude Simon, Orion aveugle : images éparses rapprochées par l'écriture}

L'illustration d'Orion aveugle de Claude Simon montre, elle aussi, la création en train de se faire, mais si l'effet de décalage est plus manifeste encore dans les relations entre le texte et les images, c'est parce que les mots « possèdent ce prodigieux pouvoir de rapprocher et de confronter ce qui, sans eux, resterait épars » (Simon 9). Parfois surprenantes au moment où elles apparaissent au lecteur, les images font partie de ces choses « éparses » rapprochées par l'écriture. Les décalages témoignent autant de l'éloignement initial des mots et des choses que de leur rapprochement, au fil de la création et de la lecture. Le passage suivant évoque le chantier de construction d'un building et le panneau publicitaire qui le présente :

Immédiatement à droite est représentée une vue en élévation du gratte-ciel tel qu'il apparaîtra une fois terminé, côte à côte avec une coupe longitudinale de l'édifice permettant de voir, comme si on en avait retiré la façade, l'intérieur divisé en casiers rectangulaires accolés et entassés les uns sur les autres. Aux divers étages, dans les diverses pièces, des hommes et des femmes se tiennent assis dans des fauteuils ou derrière des bureaux, ou debout, ou encore serrés dans des ascenseurs. (55)

Le lecteur ne peut qu'être surpris lorsqu'il découvre, à la place de la coupe longitudinale évoquée dans le texte, un montage de George Brecht intitulé Repository (56) et réalisé en 1961 (fig. 4). Il s'agit d'une étagère de bois blanc contenant des casiers, des armoires et des tiroirs. Les casiers

3 Cf. p. 100 et 135. 
pourraient pourtant rappeler les divers étages de l'immeuble à naître, séparés par des cloisons, mais ce sont des objets ordinaires qui s'y trouvent, et non des personnages représentés dans des attitudes de travail. Peut-être s'agit-il de souvenirs d'événements dignes d'être gardés en mémoire qui reposent ainsi dans les cases ? On y trouve en effet, de haut en bas, la figurine d'un motard placée devant une petite construction de bois blanc - possible miniature de l'édifice évoqué dans le texte -, une fiole en verre, un ensemble d'objets sphériques et colorés (ampoule bleue, boule de noël, balle de baseball signée, ballon blanc, doré et rouge, dont les étoiles rappellent celles du drapeau américain, pelote de fil blanc etc.). Plus bas encore, dans une petite case située entre deux portes d'armoires fermées, ont été placés un cœur rouge suspendu, sur lequel est gravé le nombre ou la date « 1944 », ainsi qu'un ensemble d'éprouvettes. Enfin, dans la partie inférieure de l'étagère et au-dessus de deux tiroirs fermés eux aussi, une dernière case sur le fond de laquelle ont été fixées une photographie et diverses clefs, contient un verre avec des brosses à dents, un petit miroir et une tomate en plastique. Cette dernière, imputrescible, semble faire de cette œuvre de Brecht une vanité moderne. Nous pourrions en effet rapprocher cette image d'une autre phrase tirée de la description du building : " tout (les peintures des murs et des machines, les meubles, les rideaux, les tissus des fauteuils, les vêtements et les visages des occupants) a un air pimpant, fonctionnel, imputrescible »(57). Gardien d'une mémoire individuelle ou collective à travers des objets devenus symboles, ce dépositaire a en effet quelque chose de «pimpant, fonctionnel, imputrescible » et semble voué à défier le passage du temps.

Pourtant, cette illustration laisse place, lorsque l'on tourne la page, à une œuvre qui semble avoir été appelée par «opposition ». Il s'agit d'un montage de Rauschenberg intitulé Canyon (fig. 5) et composé d'un grand panneau à la surface duquel ont été collés un morceau de taule ainsi que des lambeaux de tissus, d'affiches et de photographies grossièrement badigeonnés de peinture noire et blanche, et sur lequel est fixé un gros rapace noir empaillé. Ce dernier semble surgir du tableau : ses ailes sont « déployées » comme s'il planait, sa tête est tournée et l'inclinaison du cou est accentuée par un trait de peinture blanche. Loin de l'impeccable étagère de Brecht, cette seconde image, plus menaçante, répond en fait à la phrase qui poursuit le texte cité précédemment : «À la recherche sans doute de quelque charogne, de quelque cadavre putréfié d'animal, ses immenses ailes déployées, immobile et impondérable, un oiseau au plumage bleunoir se laisse porter sur l'air le long des parois glacées de la montagne ». La soudaine rupture présente dans le texte est renforcée par la maquette du livre qui place à la tourne de la page le mot « oiseau » et sur la « belle page », immédiatement visible par le lecteur, la reproduction du montage de Rauschenberg. L'image semble ainsi « aimantée » par le mot, tout comme les deux 
œuvres picturales à l'origine du texte de Claude Simon, Charlene de Rauschenberg (page de titre 16-17, 49) et Paysage avec Orion aveugle de Poussin (couverture et 130-131), ont "aimanté» et nourri l'écriture. L'illustration, ici encore, loin de témoigner d'une simple soumission de l'image au texte, réfléchit le processus créateur.

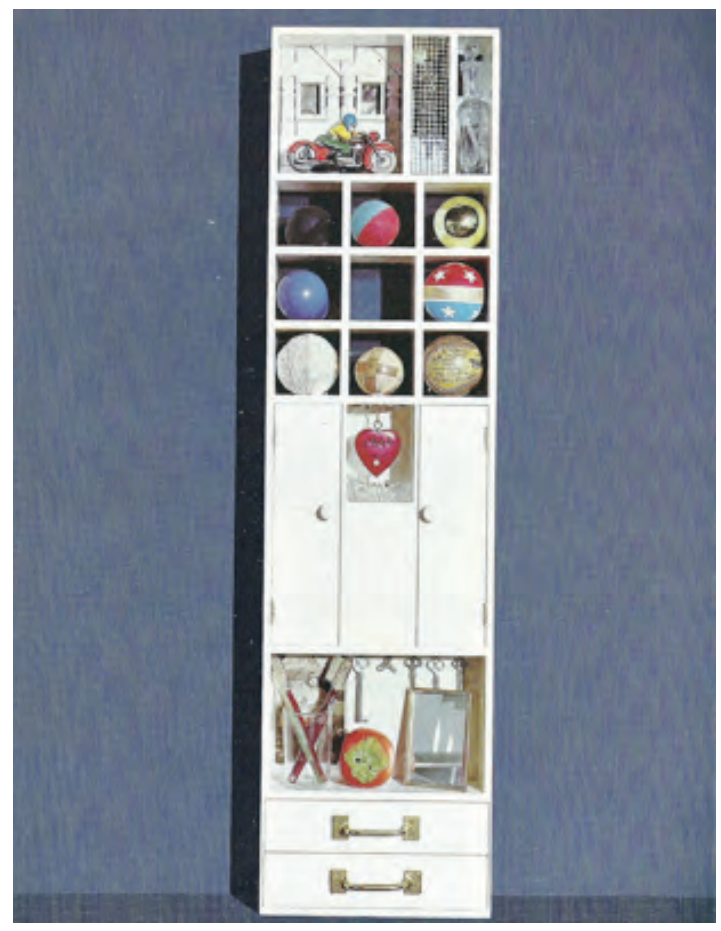

Fig. 4. BRECHT, George. Repository, 1961. Montage. $(102,5$ x 26,5 x 7,5). Museum of Modern Art, Larry Aldrich Foundation Fund, New York (photo du musée). Dans SIMON, Claude. Orion aveugle. Les sentiers de la création. Genève : Albert Skira, 1970. 56. (C) Éditions Albert Skira (C) Adagp, Paris, 2019.

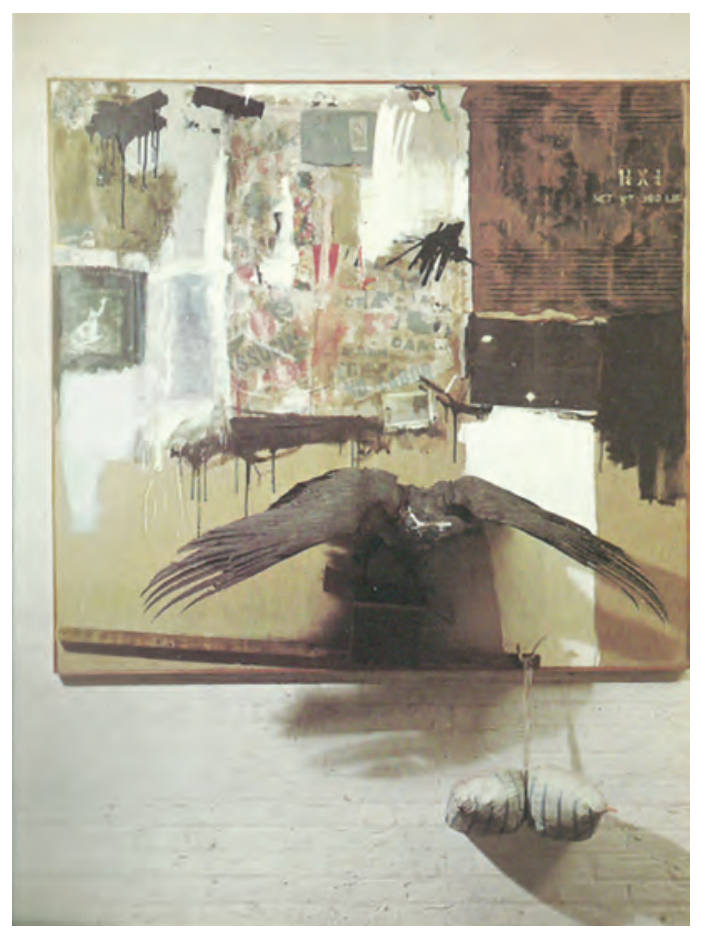

Fig. 5. RAUSCHENBERG, Robert. Canyon, 1959.

Montage. (185,5 x 167,5 x 63). Museum of Modern Art, New York. Collection Mr. And Mrs. Michael Sonnabend (photo Museum of Modern Art, New York). Dans SIMON, Claude. Orion aveugle. Les sentiers de la création. Genève : Albert Skira, 1970. 59. (C) Éditions Albert Skira (C) Robert Rauschenberg Foundation / Adagp, Paris, 2019 


\section{Interroger et représenter l'écart : seuils et vacillements}

Certaines manifestations de non subordination immédiate de l'image au texte suggèrent que la réflexion sur l'écart tient une place importante dans le cheminement créateur lui-même. On l'observe notamment, et de manières très différentes, chez Roland Barthes et Yves Bonnefoy.

\section{« Lire le recul des signes » dans les vacillements nés de l'entrelacs du texte et de l'image [Roland Barthes, L'Empire des signes]}

Dans L'Empire des signes, texte et images sont comme deux lignes autonomes qui parfois se rejoignent. Le livre débute ainsi :

Le texte ne « commente » pas les images. Les images «n'illustrent» pas le texte : chacune a été seulement pour moi le départ d'une sorte de vacillement visuel, analogue peut-être à cette perte de sens que le Zen appelle un satori; texte et images, dans leur entrelacs, veulent assurer la circulation, l'échange de ces signifiants : le corps, le visage, l'écriture, et y lire le recul des signes. (Barthes 7)

Le décalage entre texte et image rejoue le vacillement éprouvé face aux images, né de l'absence de fixité dans le rapport entre signifié et signifiant. Le signe peut alors s'ouvrir sur un autre signe. Roland Barthes voit dans la statue du moine Hôshi (fig. 6) (67) une expression de cette « fracture du signe » : son visage s'ouvre en effet étrangement sur un autre visage ${ }^{4}$ tout comme le signe ne retient pas le signifié et s'ouvre sur un autre signe.

Il peut paraître surprenant qu'un chapitre consacré à la tempura, type de friture des aliments, soit illustré par un rideau de cordons que traverse un personnage (fig. 7), reproduction de la partie droite d'un paravent. Mais il peut s'agir en fait de l'image même, quoique retardée, de la circulation des signifiants évoquée dans le préambule. De plus, ce qui lie l'image et le texte peut être l'idée de légèreté et de fragilité de la tempura que Roland Barthes apparente, par rapprochement thématique, à quelque chose qui tient « du léger, de l'aérien, de l'instantané, du fragile, du transparent du frais, du rien mais dont le vrai nom serait l'interstice sans bords pleins, ou encore : le signe vide » (38). L'image est renommée « L'interstice », qui est aussi le titre du chapitre : elle montre un espace de transition, de passage d'un lieu à un autre, suggère la possibilité d'une ouverture sur le vide. Enfin, l'impression

4 Cette statue datant de l'ère Heian (794-1185) représente le moine Baozhi d'où émerge Avalokitesvara, incarnation totalisante des onze précédents bodhisattva. 


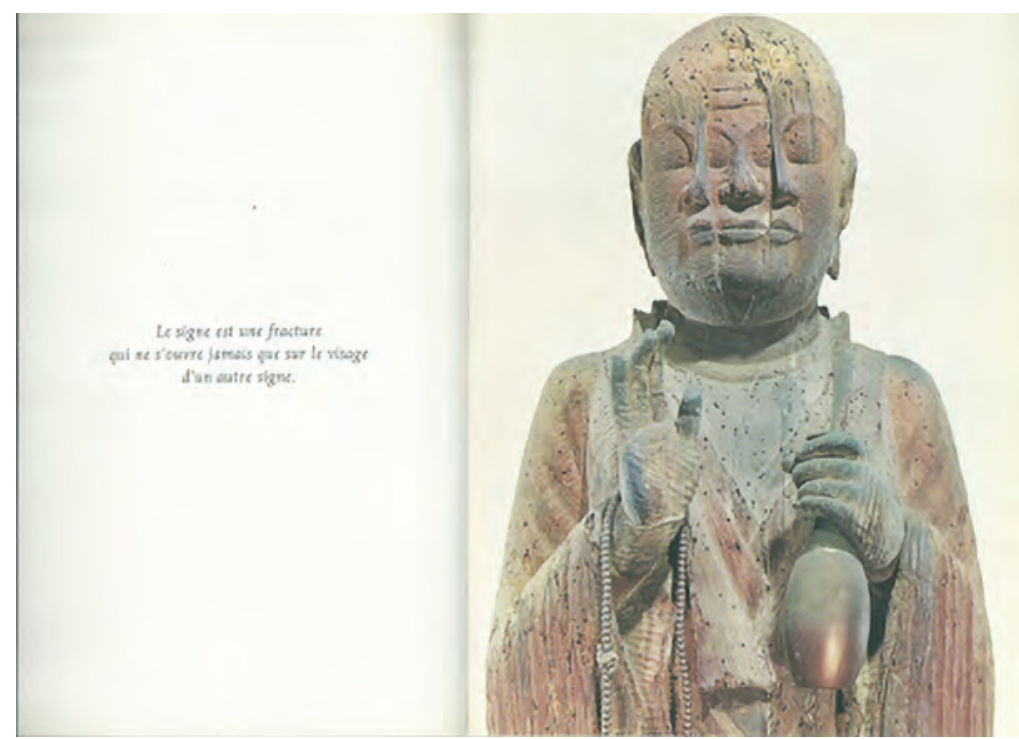

Fig. 6. Statue du moine Hôshi, fin de la période Heian. Musée National, Kyoto (photo Zauho Press, Tokyo). Dans BARTHES, Roland. L'Empire des signes. Les sentiers de la création. Genève : Albert Skira, 1970. 67. (C) Éditions du Seuil. (C) Kyoto National Museum.

« d'interstice » s'accroît par le fait que les relations entre le propos et l'image ne sont pas directement illustratives, ce qui suscite la première impression de décalage. L'ouverture verticale du rideau de cordes rappelle celle du visage du moine sur un second visage.

L'interstice, image de l'entre-deux et du « vacillement », devient ainsi un motif dans L'Empire des signes et fait l'objet de variations formelles. Selon Roland Barthes, les yeux des japonais le figurent à leur tour car " [1]'œil est plat (c'est là son miracle); ni exorbité ni renfoncé, sans bourrelet, sans poche et si l'on peut dire sans peau, il est la fente lisse d'une surface lisse » (134). On retrouve par conséquent ces yeux dans les reproductions d'un portrait photographique de l'acteur Kazuo Funaki (en ouverture et en clôture du livre) (8 et 151), d'un masque ancien de danse populaire aux yeux troués (19), des visages d'un groupe d'enfants assistant, à Tokyo, à un spectacle de « Guignol de papier » (136-137) et, pour finir, d'une photographie d'un visage prise par Nicolas Bouvier, cadrée en gros plan et accompagnée de la légende suivante : « Les yeux, et non pas le regard, la fente, et non pas l'âme » (146-147). Ces yeux, parce qu'ils semblent tracés par « un calligraphe anatomiste » qui, comme dans « la peinture alla prima, ouvre le visage d'une fente elliptique, qu'il ferme vers la tempe, d'un virage rapide de sa main » (134), rejoignent aussi le « signe ». 
Fig. 7. Le rideau de cordons (Nawa-noren) - partie droite d'un paravent, première moitié du XVIIe siècle. Encre de chine et peinture sur papier avec application de feuilles d'or, 159,6 x 90,3 cm). Collection Taki Hara, Tokyo (photo Hans-D. Weber, Cologne) (tous droits réservés). Dans BARTHES, Roland. L'Empire des signes. Les sentiers de la création. Genève : Albert Skira, 1970. 36. (C) Éditions Albert Skira.

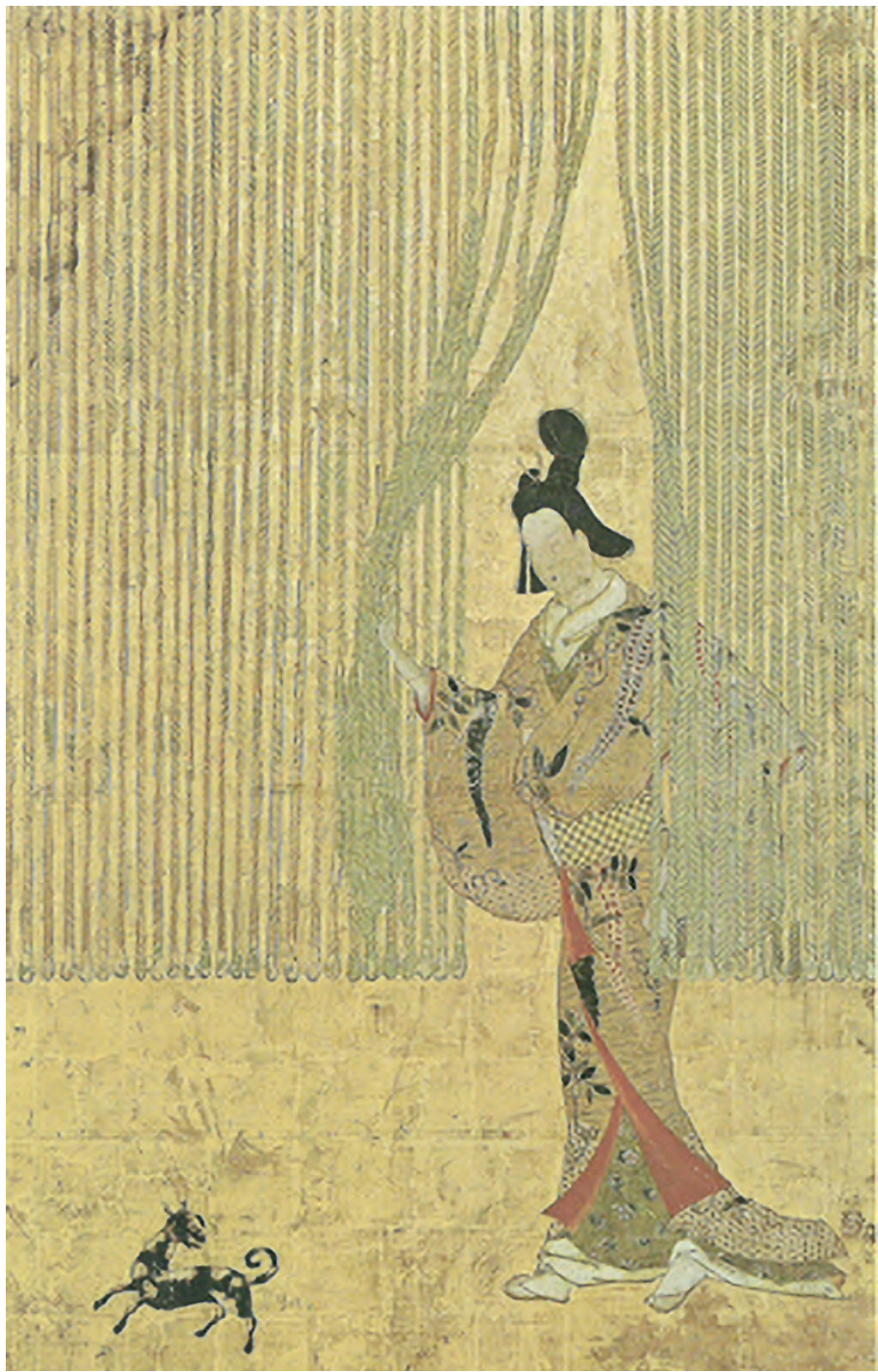


Ces rapprochements pourraient rappeler la création par analogies observée chez Octavio Paz. Pourtant, leurs approches sont essentiellement différentes. Le signe japonais ne retient pas le signifié, ne fixe pas l'essence des choses mais c'est là, justement, ce qui fait, pour Roland Barthes, sa valeur. Il n'y a pas, dans cette expérience, de « destruction » par la création ni de drame mallarméen d'une insaisissable essence. Le vide et l'écart ne constituent pas une absence mais un satori (qui est l'événement zen, une expérience de « vacillement » de la connaissance et du sujet) et s'apparentent plutôt au $M u$, signe du vide dans le bouddhisme zen également reproduit dans les premières pages du livre (12). C'est l'expression et l'expérience de ce vide singulier que fait Roland Barthes au contact de ce qu'il nomme signes.

\section{Yves Bonnefoy, L'Arrière-Pays : au « seuil » des mots et des images}

L'héritage d'Yves Bonnefoy n'est pas structuraliste et l'expérience qu'il fait de l'écart, du décalage et de l'entre-deux et dont il rend compte, lui aussi, à travers des décalages entre texte et images, est très différente, voire opposée à celle de Barthes, puisqu'il s'agit d'une expérience de l'absence. «L'Arrièrepays » est un ailleurs qui se devine ici mais en tant qu'il semble toujours échapper. C'est la promesse d'un ailleurs harmonieux, où mots et choses coïncideraient, et de la négation presque immédiate de cette promesse. Les carrefours et les seuils sont les lieux où Bonnefoy fait cette expérience car les chemins s'y rejoignent et s'y séparent, simultanément.

L'illustration singulière de l'ouvrage rejoue cette expérience à plusieurs niveaux. Des phrases tirées du texte servent toujours de légendes aux images, mais elles ne sont que rarement issues des pages qui les précèdent ou qui les suivent. La page est toujours indiquée, comme une invitation à revenir en arrière ou à sauter quelques pages. Il se crée donc un décalage et l'image semble alors illustrer deux passages à la fois. Ainsi, au moment où le détail d'une crucifixion d'Arcangelo di Cola da Camerino est reproduit (fig. 8), il illustre en quelque sorte deux passages de l'œuvre : celui dans lequel il s'insère et celui dont est tiré la légende.

Dans les pages précédant l'illustration, Yves Bonnefoy revient sur un livre qu'il avait projeté d'écrire à propos d'un voyageur qui « reparcourrait son chemin » raisonnant sur des œuvres contemplées par l'auteur lui-même en Toscane, à Arezzo (Bonnefoy 82). À la fin du texte, le voyageur devait pénétrer dans une orangerie et retrouver un inconnu rencontré quelque temps auparavant à Florence :

L'inconnu est là sur le seuil à regarder l'horizon. L'air vibre de résurrection au-dessus de la pierre sèche. (96) 
Qui est plus que lui sur le seuil? (p. 75)

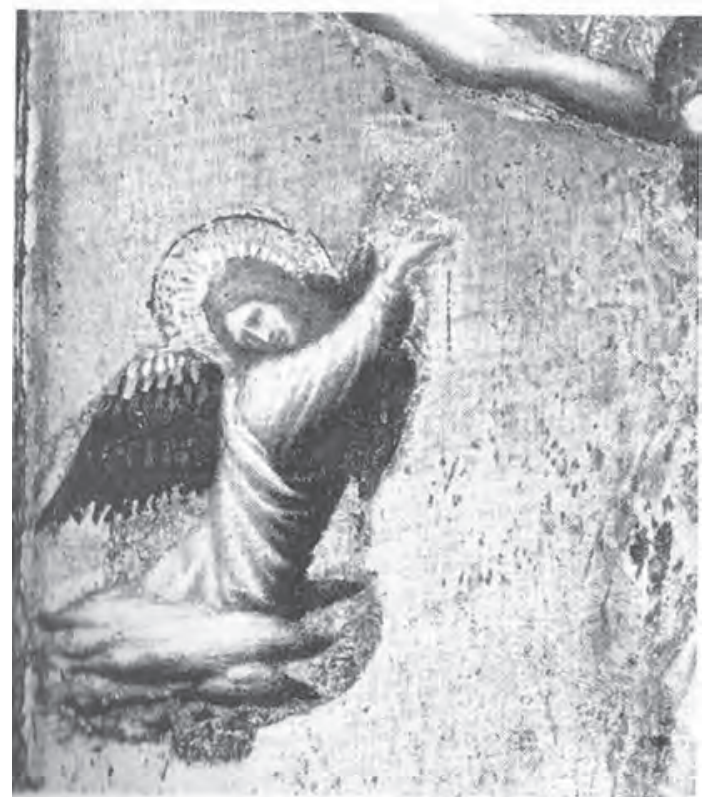

Fig. 8. Arcangelo di Cola da Camerino (actif, 1416-1429), Crucifixion, détail d'un diptyque. Huile. Collection Helen C. Frick, Pittsburgh. Dans BONNEFOY, Yves. L'Arrière-pays. Les sentiers de la création. Genève: Albert Skira, 1972. 97. (C) Éditions Gallimard. (C) Frick Art \& Historical Center, Pittsburgh.
C'est la reprise du mot « seuil », qui crée l'écho et rappelle un autre passage lu vingt pages auparavant. Il y était en effet question de la Madone d'Arcangelo di Cola di Camerino, qui compose, avec cette crucifixion, un diptyque, contemplée par Bonnefoy dans le Burlington magazine : « je me disais, qui est plus que lui sur le seuil, fautil que je parte à Camerino, pour les derniers pas sur le chemin ?» (75). L'œuvre citée dans le texte n'arrive donc que bien plus tard, retrouvée par l'imagination poétique. Le retard d'insertion de la reproduction permet de réunir ces deux passages. Mais un deuxième terme explique aussi le surgissement du souvenir de l'œuvre du florentin à cet endroit : « résurrection » (« L'air vibre de résurrection »). L'inconnu, dans le livre projeté, est au seuil de deux mondes tout comme l'est le Christ, au seuil du monde des vivants et du monde des morts, de l'Humanité et de la Divinité. Ni mort encore, ni ressuscité mais sur le point d'accomplir le verbe divin. Ce n'est pourtant pas le Christ que l'on voit dans ce détail de cette peinture, reproduite en monochrome, car on le devine seulement, en haut à droite. L'image est centrée sur un ange qui tend un calice devenu presque invisible, afin de recueillir le sang du Christ dont on distingue quelques sombres gouttes. Il détourne le regard de cet objet ainsi que de la Passion pour regarder, probablement, les hommes en contrebas. C'est ce ciboire, visible en pointillé seulement, qui révèle un sacrifice en train de s'accomplir et qui ne saurait être qu'entrevu, le spectateur voyant ce que l'ange ne peut contempler. Le décadrage permet d'insister sur ce mystérieux transfert. L'expression du visage de l'ange se substitue à celle du Christus dolens désormais hors cadre invitant ainsi à considérer un autre transfert, quant à lui irreprésentable : le sacrifice du christ, mourant pour que les hommes, à leur tour, puissent ressusciter. 
Alice Scheer: Illustrer « les sentiers de la création» : des cheminements singuliers

23

Enfin, après l'ange, le Christ et l'inconnu, c'est l'écrivain lui-même qui se trouve sur le « seuil ». Le poète, revenant sur « les sentiers de la création », retrouve alors dans ce livre consacré à un voyageur, la trace d'un précédent « roman », l'Ordalie, brûlé quelques temps auparavant. La suite du passage dit en effet :

[...] je dois reconnaître que c'est à nouveau, dans ces dédoublements, ces quatre présences [le voyageur, une femme qui se dédouble, l'inconnu] où une cinquième n'est pas sans interférer en vibrant comme l'air dehors, L'Ordalie, le « roman » que j'avais écrit puis détruit trois ou quatre années avant parce que ces bifurcations, ces décompositions prismatiques étaient certes irréductibles à toute psychologie, toute vraisemblance, se retirant comme une eau de l'écriture finie. (98)

Ce détail de la Crucifixion illustre donc non seulement et indirectement le titre de ce précédent texte, L'Ordalie (le jugement de Dieu), mais aussi le fait même d'entrevoir dans une œuvre récente, les traces d'une œuvre plus ancienne. L' " arrière-pays » d'Yves Bonnefoy n'est pas seulement un lieu idéal, un ailleurs promis $i c i$, il est peut-être aussi la manifestation dans l'œuvre présente ou reparcourue de précédents cheminements créateurs. C'est cela que permet de suggérer l'illustration « retardée».

Finalement, les auteurs évoqués dans cette étude se sont saisis de ce principe de commande original proposé par Albert Skira (mêler les mots et les images pour rendre compte de leur cheminement créateur) pour en faire un moyen d'expression complémentaire et singulier entrant en dialogue avec le texte. L'iconographie n'est, dans ces ouvrages, ni descriptive, ni redondante mais signifiante. Elle transpose la réflexion menée dans le texte, la nuance parfois, la complexifie souvent. Albert Skira était réputé pour ses illustrations narratives, surprenantes, qui dramatisent le propos. Mais les écarts observés entre le texte et l'image montrent bien que la fonction de l'illustration n'est pas, dans cette collection, uniquement " narrative » mais qu'elle prend part au cheminement créateur. Plus qu'un contrepoint et une traduction visuelle du propos développé, elle est un moyen à part entière d'interrogation et de représentation d'un cheminement créateur singulier. Et c'est souvent des décalages et des écarts entre le texte et l'image que naît cette force d'interrogation. 


\section{Ouvrages Cités}

BARTHES, Roland. L'Empire des signes. Les sentiers de la création. Genève : Albert Skira, 1970.

BONNEFOY, Yves. L'Arrière-pays. Les sentiers de la création. Genève : Albert Skira, 1972.

LE CLÉZIO, J-M. G. Haï. Les sentiers de la création. Genève : Albert Skira, 1971.

PAZ, Octavio. Le Singe grammairien. Les sentiers de la création. Genève : Albert Skira, 1972.

SIMON, Claude. Orion aveugle. Les sentiers de la création. Genève : Albert Skira, 1970.

TARDIEU, Jean. Obscurité du jour. Les sentiers de la création. Genève : Albert Skira, 1974.

\section{QEuvres CITÉES}

CORTOT, Jean. Ecritures. Toile acrylique. 1974. Collection Jean Tardieu (photo Jacqueline Hyde). Dans TARDIEU, Jean. Obscurité du jour. Les sentiers de la création. Genève : Albert Skira, 1974. 64.

MAGRITTE, René. L'Empire des lumières. Huile sur toile. 1854. Musées Royaux des Beaux-Arts de Belgique, Bruxelles (photo du musée). Dans TARDIEU, Jean. Obscurité du jour. Les sentiers de la création. Genève : Albert Skira, 1974. 17.

POUSSIN, Nicolas. Paysage avec Orion aveugle. Huile. 1658. Metropolitan Museum of Art, New York. Fletcher Fund, 1924 (photo Henry B. Beville, Alexandria, Va.). Dans SIMON, Claude. Orion aveugle. Les sentiers de la création. Genève : Albert Skira, 1970. 130-131.

Publicité pour les eaux minérales Perrier (Langelan \& Cerf, photo J. C. Dewolf), publicité pour les radiateurs en fonte Ideal Standard (Mesmer promarket, photo Mardyks), Le vieux Léon (photographie Marina Le Clézio). Dans Le Clézio, J-M. G. Haï, Les sentiers de la création. Genève : Albert Skira, 1971. 136-137.

RAUSCHENBERG, Robert. Charlene, détails. Collage. 1954. Stedelijk Museum, Amsterdam (photo du musée). Dans SIMON, Claude. Orion aveugle. Les sentiers de la création. Genève : Albert Skira, 1970. 16-17. 49.

SOJUN, Ikkyu. Calligraphie dédiée à un moineau mort. Encre sur papier. Rouleau vertical. Collection Hatakeyama, Tokyo (photo Hatakeyama). Dans TARDIEU, Jean. Obscurité du jour. Les sentiers de la création. Genève : Albert Skira, 1974. 63. 